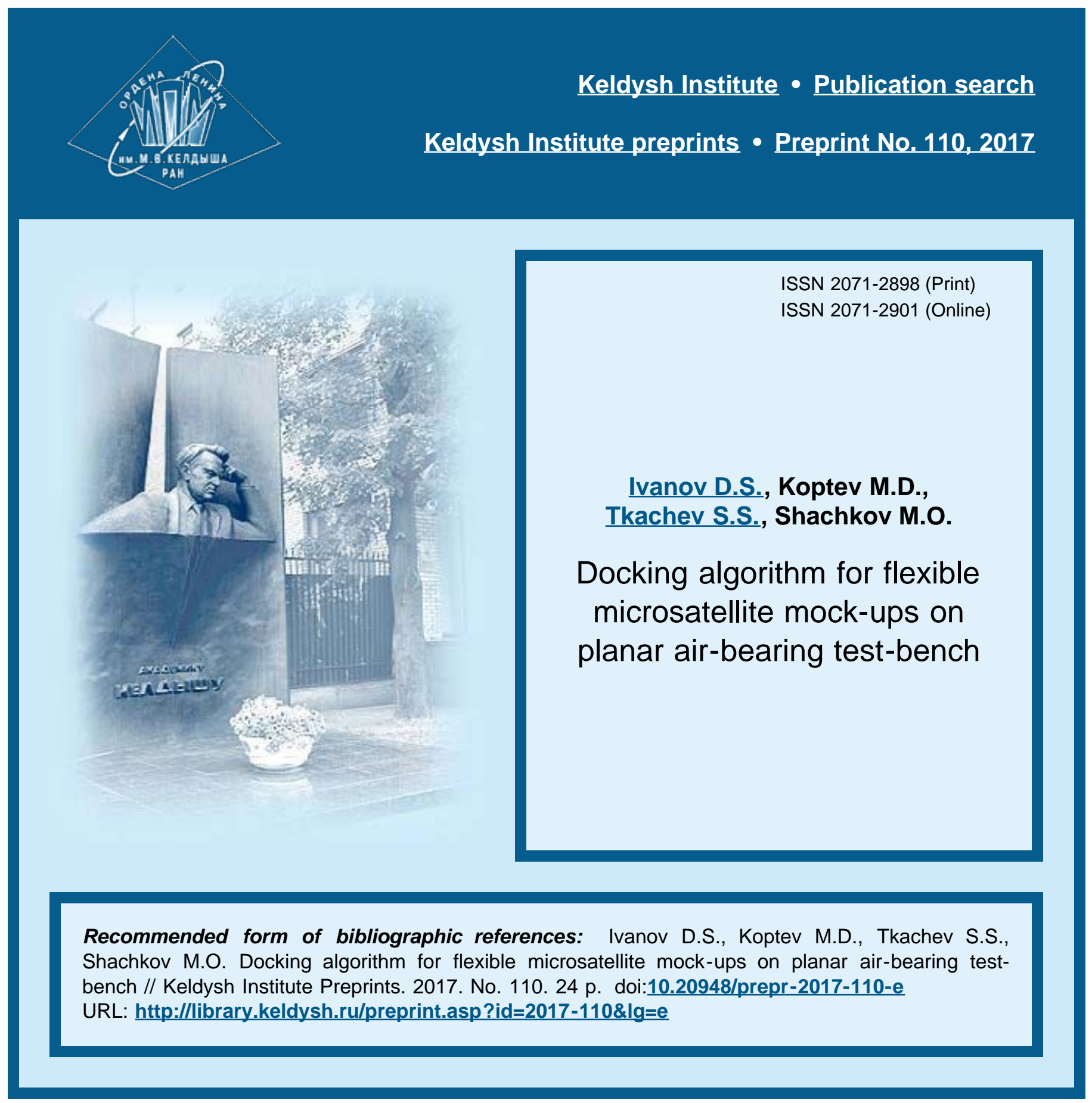




\author{
О рд н н а $Л$ н и на \\ ИНСТИТУТ ПРИКЛАДНОЙ МАТЕМАТИКИ \\ имени М.В. Келдыша \\ Р оссийской а кадемии на ук
}

D. Ivanov, M. Koptev, S. Tkachev, M. Shachkov

\title{
Docking algorithm for flexible microsatellite mock-ups on planar air-bearing test-bench
}




\section{Иванов Д.С., Коптев М.Д., Ткачев С.С., Шачков М.О.}

Стыковка макетов микроспутников с нежесткими элементами конструкции на аэродинамическом столе

В работе рассматривается алгоритм управления макетом микроспутника с гибкими стержнями во время стыковки с некооперируемой целью на аэродинамическом столе. Предложена линейная модель движения макета с нежесткими элементами. Вектор состояния корпуса макета и нежестких элементов оценивается с помощью расширенного фильтра Калмана с использованием обработки изображения. В работе представлены результаты исследования разработанных алгоритмов.

Ключевые слова: малый спутник, групповой полет, аэродинамическая сила, алгоритм управления

\section{Ivanov D., Koptev M., Tkachev S., Shachkov M.}

Docking algorithm for flexible microsatellite mock-ups on planar air-bearing test-bench

A motion control algorithm for microsatellite mock-up with flexible rods docking with noncooperative target on the air table is proposed in the paper. A linear motion model of the mock-up with flexible rods is developed. The state vector of the mock-up body and flexible rod is estimated by the extended Kalman Filter using the visual-based navigation system measurements. The results of the experimental study of the developed algorithms are presented.

Key words: test-bench, satellites docking, control algorithm, motion determination

The work supported by the Russian Science Foundation, grant № 14-11-00621. 


\section{Introduction}

Multi-satellite space systems promise a breakthrough in space exploration and scientific experiments. A group of satellites flying in small relative distances could solve ambitious tasks such as on-orbit station assembly [1], exploration of the asteroid belt [2], construction of a distribute sensing system [3] etc. The most challenging tasks for formation flying are space debris removal and satellites refueling. Both tasks require precise satellite relative motion control and docking with target that might be noncooperative. Satellite can be equipped with flexible solar panels or antennas, its oscillations can be excited by actuators control during the final phase of the docking. In this case the control algorithm should take into account the motion of flexible parts of the satellite, otherwise the oscillations could fail the docking. In the paper such an algorithm is proposed and demonstrated using laboratory facility.

The formation flying control algorithms are commonly verified on the test-bed allowing frictionless motion along the surface of smooth table. In most cases the aircushion between mock-up's legs and surface is used. Usually it is produced by the compressed air in the on-board balloons, which leads to the time-limitation of the experiments. Such facilities are widely used in research centers and universities, its overview is presented in the survey paper [4]. Another way to produce air cushion is to create airflow through the grid of holes in the flat table surface similar to the airhockey table. In this case there is no need in on-board air ballons, though there must be an air-supply system connected to the reservoir under the table surface. This kind of facility is presented in Tehnion's distributed space system laboratory [5] and in the Keldysh Institute of Applied Mathematics RAS [6].

Experiments on the air test-bed are often carried out using mock-ups with flexible elements. Testing the space manipulators [7,8], verification of algorithms for transportation of membranes by a set of flying robots for a large space station construction [9] are such examples. In [10] the problem of the attitude control for maneuvering of the mock-up with flexible structure is solved. The paper [11] considered the dynamics of the satellite with flexible space manipulator during the docking and proposed control algorithms applied either to joints of the robotic arms or to the base platform of the manipulator.

The problem of an autonomous docking between a controlled rigid spacecraft and an uncontrolled tumbling target is well studied in the literature. In the last decade significant achievements have been obtained in the field of optimal docking control strategies. In the papers $[12,13]$ the model predictive control is applied to the problem. It allows to generate the safe and fuel-optimal rendezvous trajectories that guarantee collision avoidance. Its computation requires the use of convex linear and quadratic programming. In the papers [14,15] proposed a second-order coneprogramming-based methodology to solve the rendezvous and proximity operations problem. Another approach is to apply the inverse dynamics in the virtual domain method for rapid sub-optimal docking trajectory generation. The approach imposes a polynomial shape to the both rotational and translational trajectories components of the chaser spacecraft and the optimal control problem for docking maneuvers is con- 
verted into an equivalent nonlinear programming problem having a reduced number of parameters to be optimized. The approach is applied in the paper [16] for the rapid trajectory generation algorithm for the full 6-DOF docking maneuver to an uncontrolled tumbling target. The inverse dynamics approach was implemented for CubeSats self-assembling and tested on the air table test bench [17]. Guidance strategy based on the inverse dynamics in the virtual domain and the nonlinear programming solver was applied to the path planning for docking maneuvers between two floating simulators in the Spacecraft Robotics Laboratory at the Naval Postgraduate School [18].

The fuel-optimal trajectory generation algorithms are computationally intensive, its implementation in a real-time system is very challenging. Some of them require high computational time to generate the trajectory, it does not allow onboard implementation of the algorithm in closed-loop fashion. That is why sometimes the optimal algorithms are compromised for the sake of simpler but rapid ones. The glideslope algorithm [19] is a common and widely used algorithm for trajectory planning in real time, although it disregards both optimality and path constraints. It is mainly used when a straight line approach to the target is required. Straight line approach is required often because of the line-of-sight constraints of docking navigation sensors. In the paper [20] guidance algorithm employs the glide-slope method for the rapid trajectory generation for SPHERES system. Another approach is to use the fixed precalculated reference docking trajectory, transform it into the mission plan matrix and control the satellite to achieve reference trajectory. Its application was demonstrated in the docking experiments on STEPS test-bed [21]. In the paper [22] the docking trajectory of the satellite mock-up is determined by means of polynomial expressions in time domain, its coefficients are determined by the boundary condition: initial and final state vector of the chaser mock-up. The coefficients are calculated as a function and it is easy to update it in real time onboard during the mock-up motion, however the docking trajectory is not fuel-optimal. In our paper considered the most simple approach that is similar to the one in [22]. However, it does not address the collision avoidance during docking, so we added repelled potential to generate collision avoidance force when the mock-up approaches the target not in the front of docking side.

The paper develops a control algorithm for the docking and reference trajectory tracking with consideration of the flexible motion of the rods. The camera measurements are used for estimation of the mock-up state vector. It consists of center of the mock-up body position, its velocity and deviations from the equilibrium position of two rods. The air-table itself produces disturbances caused by local nonflatness (about 3,5 $\mathrm{mm}$ for whole surface) and uneven air flow through holes. The preliminary measurements of torques and accelerations allow to develop the disturbance map of the table and to use it for prediction. To prevent the collision with target corresponding terms are included in the controller. An experimental study of the developed algorithm is presented in the paper. 


\section{Test bench COSMOS}

COSMOS (COmplex for Satellites MOtion Simulation) consists of the airbearing table, industrial fan and its control unit, air supply system and microsatellites mock-ups (Figure 1). The air bearing table includes aluminum flat perforated surface and special cavity where air is pumped.

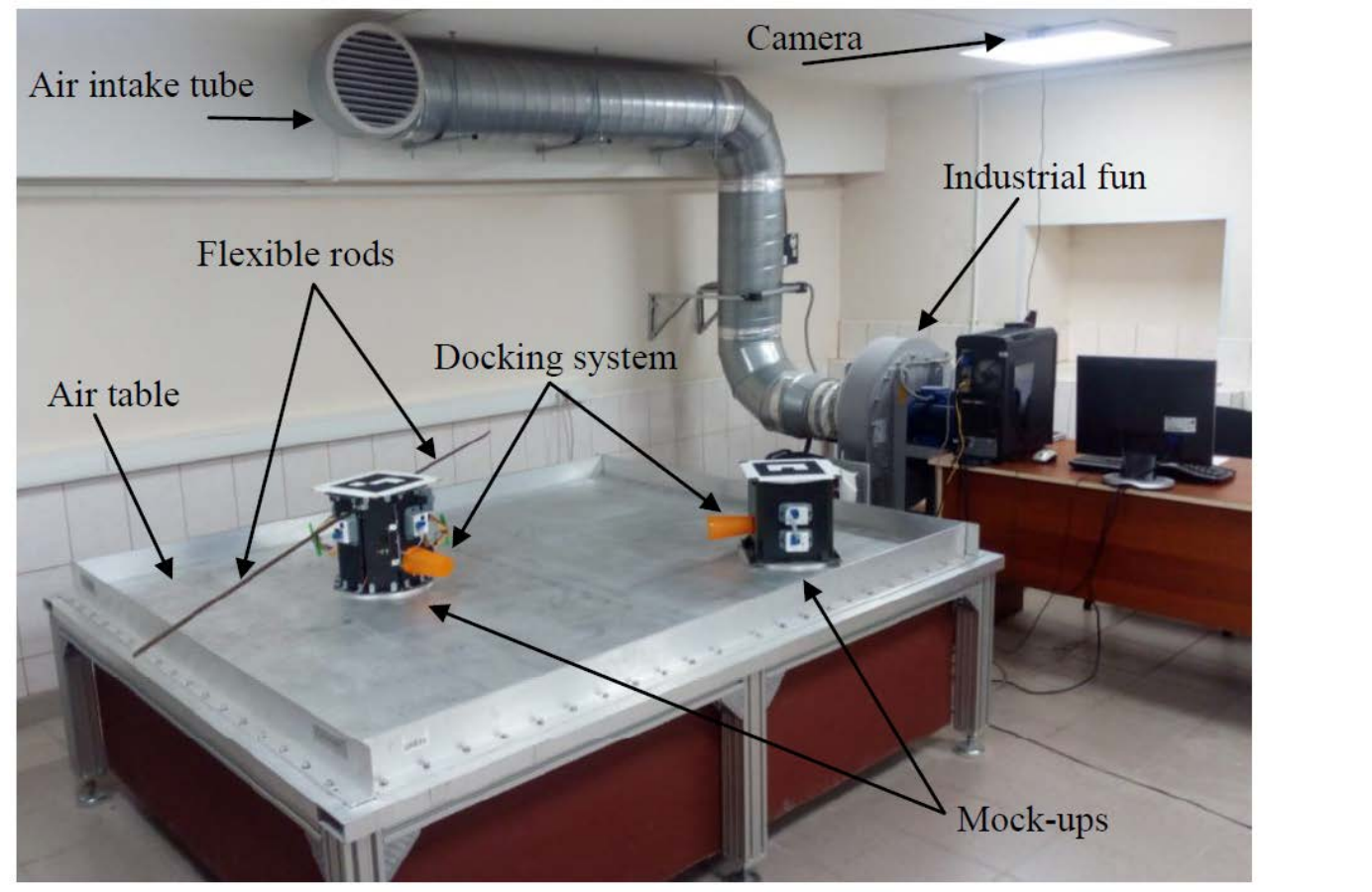

Figure 1. Laboratory facility COSMOS with flexible rods

The surface consists of the two one-cm thick plates. Plates are fixed at the bottom by the special frame that prevents bending due to the both pates weight and excessive pressure, so the result non-flatness is about $3.5 \mathrm{~mm}$. Total size of the surface is $198 \mathrm{~cm}$ by $148 \mathrm{~cm}$. The surface has the pattern of $1 \mathrm{~mm}$ diameter holes with 20 $\mathrm{mm}$ intervals. The distance between holes is chosen to provide frictionless motion for $30 \mathrm{~cm}$ platform diameter of mock-up of up to $6 \mathrm{~kg}$ mass. Mock-ups have the shape of octagonal prism of $40 \mathrm{~cm}$ height. Each side has mounting holes to install hardware both inside and outside (Figure 2). 


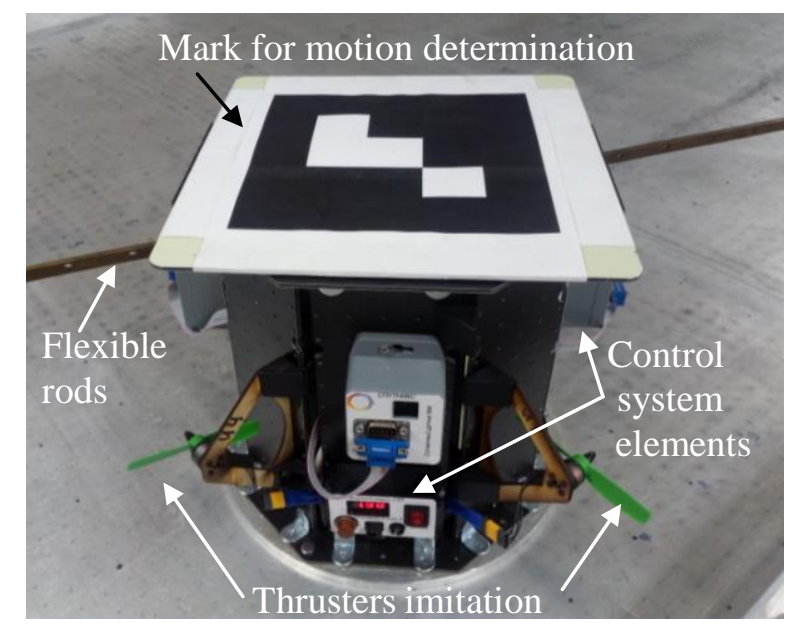

Figure 2. Satellite mock-up

The mock-up control system is based on the construction kit Orbicraft developed by SputniX Ltd [23]. Its mass is about $5.2 \mathrm{~kg}$ and axial moment of inertia is about $0.05 \mathrm{~kg} \cdot \mathrm{m}^{2}$. Control system imitator includes: on-board computer Raspberry PI 2 B; power supply system; four propellers for thruster imitation; Wi-Fi module.

There is the special mark on top of each mock-up. Marks are used for mock-up position and attitude determination using external video camera data processing [24]. These data are transmitted by Wi-Fi channel to the on-board computer which is used to calculate a control command. In case of autonomous attitude and position determination, video camera is used as independent motion determination system. Two flexible rods of $1 \mathrm{~m}$ length each are attached to the mock-up body. During the control phase the rods oscillations excites. Along with aerodynamic and gravitational disturbances it leads to unacceptable errors for docking with noncooperative target.

\section{Motion model of the mock-up with flexible rods}

The mock-up with two flexible rods is considered. Let the rods be fixed at one end on the mock-up body (see Figure 3). General motion equation derivation for a satellite with flexible elements one can find in [25-27]. The nonlinear equations are too balky and not convenient for development of the control algorithm. That is why only the linear equations are presented in the paper. It describes the satellite motion rather well under the assumption of small flexible elements deformations.

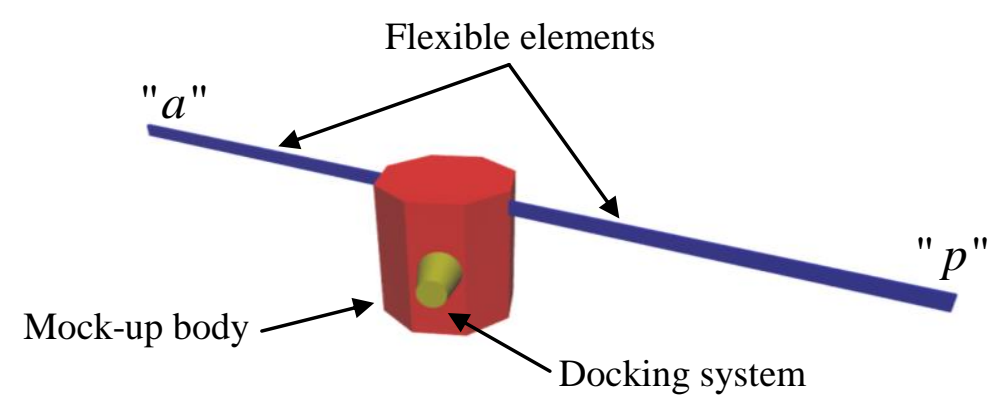

Figure 3. Scheme of the mock-up with flexible rods 


\subsection{Mock-up motion equations derivation}

Displacements of the $i$-th rod point due to the deformations are written as follows

$$
\mathbf{u}_{i}=\mathbf{a}_{i 1} q_{1}+\mathbf{a}_{i 2} q_{2}+\ldots=\mathbf{A}_{i} \mathbf{q}
$$

where $\mathbf{a}_{i j}$ is a displacement vector due to $j$-th deformation mode and $q_{j}$ are the amplitudes of these modes. Linearized equations of the angular and flexible motion of the satellite with two rods are (see paper [27])

$$
\mathbf{S}\left(\begin{array}{c}
\dot{\boldsymbol{\omega}} \\
\ddot{\mathbf{q}}_{a} \\
\ddot{\mathbf{q}}_{p}
\end{array}\right)=\left(\begin{array}{c}
\mathbf{T}_{s}-\mathbf{f}_{\omega a}-\mathbf{f}_{\omega p} \\
-\mathbf{f}_{a} \\
-\mathbf{f}_{p}
\end{array}\right) .
$$

Here $\boldsymbol{\omega}$ is the angular velocity of the satellite body, $\mathbf{q}_{a}, \mathbf{q}_{p}$ are the vectors of mode amplitudes of two rods, subscripts " $a$ " and " $p$ " are chosen to indicate the difference between two rods: " $a$ " for conditional "antenna, " $p$ " for conditional "panel" (see Figure 3),

$$
\begin{aligned}
\mathbf{S}= & \left(\begin{array}{ccc}
\mathbf{J} & \mathbf{S}_{\omega a} & \mathbf{S}_{\omega p} \\
\mathbf{S}_{\omega a}^{T} & \mathbf{M}_{a} & \mathbf{S}_{a p} \\
\mathbf{S}_{\omega p}^{T} & \mathbf{S}_{a p}^{T} & \mathbf{M}_{p}
\end{array}\right), \\
\mathbf{J}= & \mathbf{J}_{s}+\mathbf{J}_{a}+m_{a} \mathbf{K}\left(\mathbf{a}, \mathbf{a}_{1}\right)+m_{a} \mathbf{K}\left(\mathbf{a}_{1}, \mathbf{a}_{2}\right)+\mathbf{J}_{p}+m_{p} \mathbf{K}\left(\mathbf{p}, \mathbf{p}_{1}\right)+ \\
& +m_{p} \mathbf{K}\left(\mathbf{p}_{1}, \mathbf{p}_{2}\right)-\frac{1}{m} \mathbf{K}\left(m_{a} \mathbf{a}+m_{p} \mathbf{p}, m_{a} \mathbf{a}+m_{p} \mathbf{p}\right),
\end{aligned}
$$

$\mathbf{J}_{s}, \mathbf{J}_{a}, \mathbf{J}_{p}$ are the inertia tensors of mockup body, and the two rods respectively, $m_{a}$, $m_{p}$ are the rods masses, $\mathbf{a}_{1}, \mathbf{p}_{1}$ are the vectors from mockup body center of mass to the rods attachment points, $\mathbf{a}_{2}, \mathbf{p}_{2}$ are the vectors from attachment point to the center of mass of the undeformed rods. $\mathbf{K}(\mathbf{x}, \mathbf{y})$ is the following matrix

$$
\begin{aligned}
& \mathbf{K}(\mathbf{x}, \mathbf{y})=\left(\begin{array}{ccc}
x_{2} y_{2}+x_{3} y_{3} & -x_{2} y_{1} & -x_{3} y_{1} \\
-x_{1} y_{2} & x_{1} y_{1}+x_{3} y_{3} & -x_{3} y_{2} \\
-x_{1} y_{3} & -x_{2} y_{3} & x_{1} y_{1}+x_{2} y_{2}
\end{array}\right) \\
& \mathbf{S}_{\omega a}=\sum_{i} m_{a i}\left(\mathbf{a}_{1}+\mathbf{r}_{a i}\right) \times \mathbf{A}_{a i}-\frac{1}{m}\left(m_{a} \mathbf{a}+m_{p} \mathbf{p}\right) \times \mathbf{A}_{a}, \mathbf{A}_{a}=\sum_{i} m_{a i} \mathbf{A}_{a i}, \\
& \mathbf{S}_{\omega p}=\sum_{i} m_{p i}\left(\mathbf{p}_{1}+\mathbf{r}_{p i}\right) \times \mathbf{A}_{p i}-\frac{1}{m}\left(m_{a} \mathbf{a}+m_{p} \mathbf{p}\right) \times \mathbf{A}_{p}, \mathbf{A}_{p}=\sum_{i} m_{p i} \mathbf{A}_{p i},
\end{aligned}
$$

$\mathbf{M}_{a}=\sum_{i} m_{a i} \mathbf{A}_{a i}^{T} \mathbf{A}_{a i}-\frac{1}{m} \mathbf{A}_{a}^{T} \mathbf{A}_{a}$ (in case of normalized modes $\mathbf{M}_{a}=\mathbf{E}-\frac{1}{m} \mathbf{A}_{a}^{T} \mathbf{A}_{a}, \mathbf{E}$ is the identity matrix, its size corresponds to the number of modes taken into account), 


$$
\begin{aligned}
& \mathbf{S}_{a p}=-\frac{1}{m} \mathbf{A}_{a}^{T} \mathbf{A}_{p}, \mathbf{M}_{p}=\sum_{i} m_{p i} \mathbf{A}_{p i}^{T} \mathbf{A}_{p i}-\frac{1}{m} \mathbf{A}_{p}^{T} \mathbf{A}_{p}, \\
& \mathbf{f}_{\omega a}=\mathbf{a} \times m_{a}\left(\frac{\mathbf{F}_{O}}{m}-\frac{\mathbf{F}_{a}}{m_{a}}\right)+\mathbf{a}_{2} \times \mathbf{F}_{a}-\mathbf{T}_{a}, \\
& \mathbf{f}_{\omega p}=\mathbf{p} \times m_{p}\left(\frac{\mathbf{F}_{O}}{m}-\frac{\mathbf{F}_{p}}{m_{p}}\right)+\mathbf{p}_{2} \times \mathbf{F}_{p}-\mathbf{T}_{p}, \\
& \mathbf{f}_{a}=\sum_{i}\left(m_{a i} \mathbf{A}_{a i}^{T}\left(\frac{\mathbf{F}_{O}}{m}-\frac{\mathbf{F}_{a i}}{m_{a i}}\right)-m_{a i} \mathbf{A}_{a i}^{T} \mathbf{A}_{a i} \mathbf{\Omega}_{a} \mathbf{q}_{a}\right), \\
& \mathbf{f}_{p}=\sum_{i}\left(m_{p i} \mathbf{A}_{p i}^{T}\left(\frac{\mathbf{F}_{O}}{m}-\frac{\mathbf{F}_{p i}}{m_{p i}}\right)-m_{p i} \mathbf{A}_{p i}^{T} \mathbf{A}_{p i} \mathbf{\Omega}_{p} \mathbf{q}_{p}\right),
\end{aligned}
$$

$\mathbf{F}_{O}, \mathbf{F}_{a}, \mathbf{F}_{p}$ are the vectors of all external forces acting upon whole mock-up and rods alone respectively $\mathbf{T}_{s}, \mathbf{T}_{a}, \mathbf{T}_{p}$ are the torques acting upon mockup body and rods, $\boldsymbol{\Omega}_{a}, \boldsymbol{\Omega}_{b}$ are the matrices of eigen frequencies: $\boldsymbol{\Omega}=\operatorname{diag}\left(\begin{array}{lll}\omega_{1}^{2} & \omega_{2}^{2} & \ldots\end{array}\right)$. Values $m_{a i}$, $m_{p i}, \mathbf{r}_{a i}, \mathbf{r}_{p i}, \mathbf{A}_{a i}, \mathbf{A}_{p i}$ one can determine using finite element method.

In case when there are no torques and forces acting upon rods $\mathbf{F}_{a}=\mathbf{F}_{b}=0$, $\mathbf{T}_{p}=\mathbf{T}_{a}=0, \mathbf{F}_{a i}=\mathbf{F}_{b i}=0$. In addition, two identical rods and its symmetrical attachment are considered. In this case

$$
\begin{aligned}
& \mathbf{A}_{a}=-\mathbf{A}_{b}=\mathbf{A}, m_{a}=m_{b}=m_{l}, \mathbf{a}_{1}=-\mathbf{b}_{1}=\mathbf{d}_{1}, \mathbf{a}=-\mathbf{b}=\mathbf{d}, \\
& \mathbf{J}_{a}=\mathbf{J}_{b}=\mathbf{J}_{d}, \\
& \mathbf{S}=\left(\begin{array}{ccc}
\mathbf{J} & \mathbf{S}_{\omega} & \mathbf{S}_{\omega} \\
\mathbf{S}_{\omega}^{T} & \mathbf{M} & \mathbf{S}_{d} \\
\mathbf{S}_{\omega}^{T} & \mathbf{S}_{d}^{T} & \mathbf{M}
\end{array}\right), \\
& \mathbf{S}_{\omega a}=\sum_{i} m_{a i}\left(\mathbf{a}_{1}+\mathbf{r}_{a i}\right) \times \mathbf{A}_{a i}=\mathbf{S}_{\omega p}=\mathbf{S}_{\omega}, \\
& \mathbf{J}=\mathbf{J}_{s}+2 \mathbf{J}_{d}+2 m_{d} \mathbf{K}\left(\mathbf{d}, \mathbf{d}_{1}\right)+2 m_{d} \mathbf{K}\left(\mathbf{d}_{1}, \mathbf{d}_{2}\right), \\
& \mathbf{M}=\mathbf{E}-\frac{1}{m} \mathbf{A}^{T} \mathbf{A}, \mathbf{S}_{l}=-\frac{1}{m} \mathbf{A}^{T} \mathbf{A}, \\
& \mathbf{f}_{\omega a}=\frac{m_{d}}{m} \mathbf{d} \times \mathbf{F}_{O}, \mathbf{f}_{\omega p}=-\frac{m_{d}}{m} \mathbf{d} \times \mathbf{F}_{O}, \mathbf{f}_{a}=\mathbf{A}^{T} \frac{\mathbf{F}_{O}}{m}-\mathbf{\Omega} \mathbf{q}_{a}, \mathbf{f}_{p}=-\mathbf{A}^{T} \frac{\mathbf{F}_{O}}{m}-\mathbf{\Omega} \mathbf{q}_{p} .
\end{aligned}
$$

So, the Eqs. (2) are simplified to a form

$$
\begin{aligned}
\mathbf{J} \dot{\boldsymbol{\omega}}+\mathbf{S}_{\omega} \ddot{\mathbf{q}}_{a}+\mathbf{S}_{\omega} \ddot{\mathbf{q}}_{p}=\mathbf{T}_{s}, \\
\mathbf{S}_{\omega}^{T} \dot{\boldsymbol{\omega}}+\mathbf{M} \ddot{\mathbf{q}}_{a}+\mathbf{S}_{l} \ddot{\mathbf{q}}_{p}=\mathbf{A}^{T} \frac{\mathbf{F}_{O}}{m}-\mathbf{\Omega} \mathbf{q}_{a},
\end{aligned}
$$




$$
\mathbf{S}_{\omega}^{T} \dot{\mathbf{\omega}}+\mathbf{S}_{l}^{T} \ddot{\mathbf{q}}_{a}+\mathbf{M} \ddot{\mathbf{q}}_{p}=-\mathbf{A}^{T} \frac{\mathbf{F}_{O}}{m}-\mathbf{\Omega} \mathbf{q}_{p} .
$$

Finite element model has shown that it is reasonable to take into account only one mode for each rod. Consider planar motion only. In this case $\mathbf{A}$ is a vector and it can be written as follows

$$
\mathbf{A}=\left(\begin{array}{l}
A_{1} \\
A_{2}
\end{array}\right), \mathbf{A}^{T} \mathbf{A}=A_{1}^{2}+A_{2}^{2}=A^{2} .
$$

In the case of planar motion only the vectors and matrices $\mathbf{S}_{\omega}=S_{\omega}, \boldsymbol{\omega}=\omega, \mathbf{J}=J$, $\mathbf{q}_{a}=q_{a}, \mathbf{q}_{p}=q_{p} \mathbf{\Omega}=\Omega, \mathbf{T}_{s}=T_{s}$ are just scalars.

Finally, the angular and flexible motion equations has a form:

$$
\begin{gathered}
J \dot{\omega}+S_{\omega} \ddot{q}_{a}+S_{\omega} \ddot{q}_{p}=T_{s}, \\
S_{\omega} \dot{\omega}+\left(1-\frac{1}{m} A^{2}\right) \ddot{q}_{a}-\frac{1}{m} A^{2} \ddot{q}_{p}=\mathbf{A}^{T} \frac{\mathbf{F}_{O}}{m}-\Omega q_{a}, \\
S_{\omega} \dot{\omega}-\frac{1}{m} A^{2} \ddot{q}_{a}+\left(1-\frac{1}{m} A^{2}\right) \ddot{q}_{p}=-\mathbf{A}^{T} \frac{\mathbf{F}_{O}}{m}-\Omega q_{p} .
\end{gathered}
$$

Rewrite it in the matrix-vector form

$$
\mathbf{S}\left(\begin{array}{c}
\dot{\omega} \\
\ddot{q}_{a} \\
\ddot{q}_{p}
\end{array}\right)=\mathbf{B}_{f}\left(\begin{array}{c}
F_{x} \\
F_{y} \\
T_{s}
\end{array}\right)+\mathbf{C}_{f}\left(\begin{array}{c}
\varphi \\
q_{a} \\
q_{p}
\end{array}\right),
$$

where

$$
\mathbf{S}=\left[\begin{array}{ccc}
J & S_{\omega} & S_{\omega} \\
S_{\omega} & \left(1-A^{2} / m\right) & A^{2} / m \\
S_{\omega} & A^{2} / m & \left(1-A^{2} / m\right)
\end{array}\right], \mathbf{B}_{f}=\left[\begin{array}{ccc}
0 & 0 & 1 \\
-A_{1} / m & -A_{2} / m & 0 \\
A_{1} / m & A_{2} / m & 0
\end{array}\right], \mathbf{C}_{f}=\left[\begin{array}{ccc}
0 & 0 & 0 \\
0 & \Omega & 0 \\
0 & 0 & \Omega
\end{array}\right] .
$$

Mock-up center of mass $\mathbf{r}=\left[\begin{array}{ll}x & y\end{array}\right]^{T}$ motion is described by

$\ddot{\mathbf{r}}=\mathbf{F}_{O} / m$.

Mock-up body center of mass position is determined by

$$
\mathbf{r}_{s}=\mathbf{r}+\frac{1}{m} \mathbf{A}\left(q_{p}-q_{a}\right) \text {. }
$$

Eqs (3) and (4) supplemented by kinematic equations will be used for further analysis of the system dynamics.

Consider the state vector of the planar motion of the mock-up with flexible rods as follows

$$
\mathbf{x}=\left[\begin{array}{llllllllll}
x & y & \varphi & q_{a} & q_{p} & \dot{x} & \dot{y} & \dot{\varphi} & \dot{q}_{a} & \dot{q}_{p}
\end{array}\right]^{T} .
$$

Rewrite the Eqs. (3) and (4) in the standard form:

$$
\dot{\mathbf{x}}=\mathbf{F x}+\mathbf{B u}
$$


where

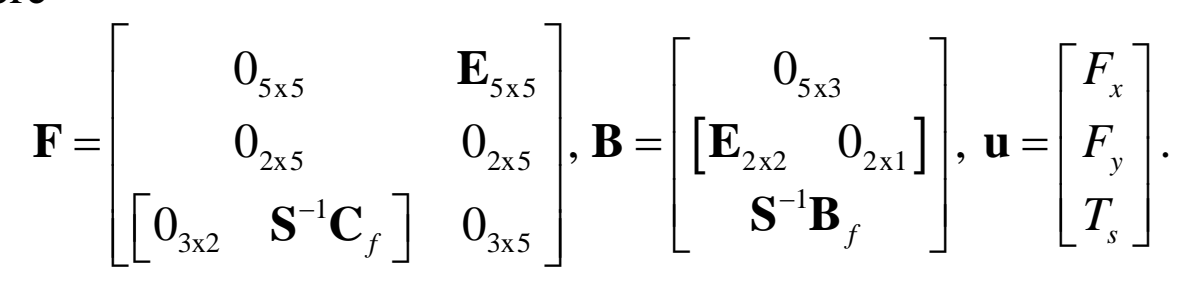

\subsection{Control synthesis}

Mock-up body center of mass motion can be derived from Eqs. (4) and (5) and it is described by the following equation in the linear case

$$
m \ddot{\mathbf{r}}_{s}=\mathbf{F}_{O}+\mathbf{A}\left(\ddot{q}_{b}-\ddot{q}_{a}\right) \text {. }
$$

Consider the control that guarantees

$$
\begin{aligned}
& J \dot{\omega}=-k_{\omega} \Delta \omega-k_{\varphi} \Delta \varphi+J \dot{\omega}_{r e f}, \\
& \ddot{\mathbf{r}}_{s}=-k_{v} \Delta \dot{\mathbf{r}}_{s}-k_{r} \Delta \mathbf{r}_{s}+\ddot{\mathbf{r}}_{r e f}
\end{aligned}
$$

where $\Delta \mathbf{r}_{s}=\mathbf{r}_{s}-\mathbf{r}_{r e f}, \Delta \dot{\mathbf{r}}_{s}=\dot{\mathbf{r}}_{s}-\dot{\mathbf{r}}_{r e f}, \Delta \omega=\omega-\omega_{r e f}, \Delta \varphi=\varphi-\varphi_{r e f}$, subscript "ref" means reference trajectory, $k_{\varphi}, k_{\omega}, k_{r}, k_{v}$ are positive constants. Thus, we demand that the motion of the mock-up body tracks the certain reference trajectory. To find this control one need to exclude $\ddot{\mathbf{q}}=\left[\ddot{q}_{a} \ddot{q}_{p}\right]^{T}$ from Eqs. (3) and (7):

$$
\begin{aligned}
& \ddot{\mathbf{q}}=\left(\mathbf{M}_{q}-\mathbf{S}_{\omega}^{T} J^{-1} \mathbf{S}_{\omega}\right)^{-1}\left(-\mathbf{C}_{\omega} \mathbf{q}+\mathbf{B}_{r} \mathbf{F}_{O}-\mathbf{S}_{\omega}^{T} J^{-1} T_{s}\right), \\
& J \dot{\omega}+\mathbf{S}_{\omega}\left(\mathbf{M}_{q}-\mathbf{S}_{\omega}^{T} J^{-1} \mathbf{S}_{\omega}\right)^{-1}\left(-\mathbf{C}_{\omega} \mathbf{q}+\mathbf{B}_{r} \mathbf{F}_{O}-\mathbf{S}_{\omega}^{T} J^{-1} T_{s}\right)=T_{s}, \\
& \ddot{\mathbf{r}}_{s}=\mathbf{F}_{O} / m-\mathbf{B}_{r}^{T}\left(\mathbf{M}_{q}-\mathbf{S}_{\omega}^{T} \mathbf{J}^{-1} \mathbf{S}_{\omega}\right)^{-1}\left(-\mathbf{C}_{\omega} \mathbf{q}+\mathbf{B}_{r} \mathbf{F}_{O}-\mathbf{S}_{\omega}^{T} J^{-1} T_{s}\right) .
\end{aligned}
$$

Here

$$
\mathbf{S}_{\omega}=\left[\begin{array}{ll}
S_{\omega} & S_{\omega}
\end{array}\right], \mathbf{M}_{q}=\left(\begin{array}{cc}
1-\frac{1}{m} A^{2} & \frac{1}{m} A^{2} \\
\frac{1}{m} A^{2} & 1-\frac{1}{m} A^{2}
\end{array}\right), \mathbf{C}_{\omega}=\left(\begin{array}{cc}
\omega_{0}^{2} & 0 \\
0 & \omega_{0}^{2}
\end{array}\right), \mathbf{B}_{r}=\frac{1}{m}\left(\begin{array}{c}
\mathbf{A}^{T} \\
-\mathbf{A}^{T}
\end{array}\right) .
$$

Taking into account (8) the following control law can be obtained $\mathbf{u}=\mathbf{U}^{-1} \mathbf{b}$,

$$
\begin{aligned}
\mathbf{U} & =\left(\begin{array}{cc}
\frac{1}{m} \mathbf{E}_{2 x 2}-\mathbf{B}_{r}^{T}\left(\mathbf{M}_{q}-\mathbf{S}_{\omega}^{T} J^{-1} \mathbf{S}_{\omega}\right)^{-1} \mathbf{B}_{r} & \mathbf{B}_{r}^{T}\left(\mathbf{M}_{q}-\mathbf{S}_{\omega}^{T} J^{-1} \mathbf{S}_{\omega}\right)^{-1} \mathbf{S}_{\omega}^{T} J^{-1} \\
-\mathbf{S}_{\omega}^{T}\left(\mathbf{M}_{q}-\mathbf{S}_{\omega}^{T} J^{-1} \mathbf{S}_{\omega}\right)^{-1} \mathbf{B}_{r} & 1+\mathbf{S}_{\omega}\left(\mathbf{M}_{q}-\mathbf{S}_{\omega}^{T} J^{-1} \mathbf{S}_{\omega}\right)^{-1} \mathbf{S}_{\omega}^{T} J^{-1}
\end{array}\right), \\
\mathbf{b} & =\left(\begin{array}{c}
-k_{v} \Delta \dot{\mathbf{r}}-k_{r} \Delta \mathbf{r}+\ddot{\mathbf{r}}_{r e f}-\mathbf{B}_{r}^{T}\left(\mathbf{M}_{q}-\mathbf{S}_{\omega}^{T} J^{-1} \mathbf{S}_{\omega}\right)^{-1} \mathbf{C}_{\omega} \mathbf{q} \\
-k_{\omega} \Delta \omega-k_{\varphi} \Delta \varphi+J \dot{\omega}_{r e f}-\mathbf{S}_{\omega}\left(\mathbf{M}_{q}-\mathbf{S}_{\omega}^{T} J^{-1} \mathbf{S}_{\omega}\right)^{-1} \mathbf{C}_{\omega} \mathbf{q}
\end{array}\right)
\end{aligned}
$$


Thus, the control law in Eq. (9) takes into account current deviations of the flexible elements $\mathbf{q}$ to produce the control force for tracking of mock-up body along the reference trajectory. The control law eliminates the oscillations of the mock-up position and attitude caused by rods vibrations. However, in practice the control force is limited, so the actuators could not accurately perform the control commands, the oscillations of the mock-up body remains, but nevertheless it amplitude decreases in time.

\subsection{Control realization by fans}

The control commands calculated using Eq. (9) are finally executed by four fans are installed on board in the configuration, shown in Figure 4. Each ventilator is unidirectional, i.e. it produces the force in one direction only. The control vector $\mathbf{u}$ has three components while we have four forces to produce it. So, one need to solve the task of the calculation of the required thrusts of the fans.

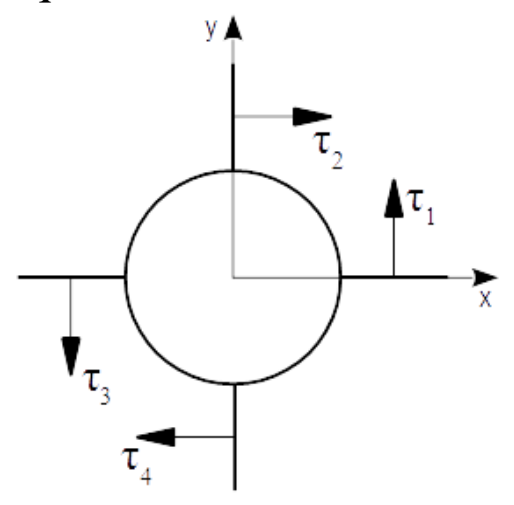

Figure 4. Scheme of ventilators placement

The implementation of the torque and force by the fans' thrust is as follows

$$
\sum_{i=1}^{4} \boldsymbol{\tau}_{i}=\mathbf{F}_{O}, \sum_{i=1}^{4} \mathbf{l}_{i} \times \boldsymbol{\tau}_{i}=\mathbf{T}_{s},
$$

where $\mathbf{F}_{O}=\left[\begin{array}{ll}F_{x} & F_{y}\end{array}\right]^{T}$ is a calculated control force, $\mathbf{T}_{s}=\left[\begin{array}{lll}0 & 0 & T_{s}\end{array}\right]^{T}$ is a calculated control torque, $\boldsymbol{\tau}_{i}$ are the thrust of fans, $\mathbf{l}$ is the vector of thrust application. In scalar form (see Figure 4)

$$
\tau_{2}-\tau_{4}=F_{x}, \tau_{1}-\tau_{3}=F_{y}, l\left(\tau_{1}-\tau_{2}+\tau_{3}-\tau_{4}\right)=T_{s},
$$

The fans rotate in only one direction, thus the restrictions on sign of $\tau_{i}$ are $\tau_{i} \geq 0, i=\overline{1,4}$. Let us minimize the function

$\Phi=\tau_{1}^{2}+\tau_{2}^{2}+\tau_{3}^{2}+\tau_{4}^{2} \rightarrow \min$.

In the paper [6] the minimization task is solved. It is shown that for any values of $F_{x}, F_{y}$ и $T_{s}$ the solution exists and has the form:

$$
\tau_{1}=0.25\left(T_{s} / l+2 F_{y}\right)+\Delta, \tau_{2}=-0.25\left(T_{s} / l-2 F_{x}\right)+\Delta,
$$




$$
\tau_{3}=0.25\left(T_{s} / l-2 F_{y}\right)+\Delta, \tau_{4}=-0.25\left(T_{s} / l+F_{x}\right)+\Delta,
$$

where

$$
\Delta=\left|\min \left(0.25\left(T_{s} / l+2 F_{y}\right) \quad-0.25\left(T_{s} / l-2 F_{x}\right) \quad 0.25\left(T_{s} / l-2 F_{y}\right) \quad-0.25\left(T_{s} / l+2 F_{x}\right)\right)\right| .
$$

The calculated control is converted into control commands based on the calibration results using the fan motor model described in [28]. Note, that real fans has restrictions on produced thrust, i.e. $\tau_{i} \in\left[0 ; \tau_{\max }\right], i=\overline{1,4}$, where $\tau_{\max }>0$ is the maximum possible thrust. In the case, one of the $\tau_{k}$ calculated using Eq. (10) exceeds value $\tau_{\max }$, all the thrusts should be proportionally decreased to avoid the saturation. Thus, in that case the control $\mathbf{u}$ calculated using Eq. (9) is performed with error due to fans force constrains. The situation could be prevented by choosing corresponding control parameters in Eq. (9), but in real experiments with mock-ups motion affected by the air table disturbances, slight delay of fans response etc. it is not simple to avoid the fans saturation.

\section{Motion determination algorithm}

Extended Kalman Filter (EKF) is a well-known and well-established for aerospace applications algorithm. It is characterized by a relatively low computational cost and allows to estimate non-measured parts of the state vector. Let us apply it for mock-up attitude and flexible rods motion determination in the case of available measurements of the mock-up body position and attitude.

\subsection{Kalman filter basics}

Kalman filter is a recursive algorithm that uses dynamical system model and sensor readings for actual motion reconstruction. State vector assumption $\hat{\mathbf{x}}_{k-1}^{+}=\hat{\mathbf{x}}\left(t_{k}\right)$ is calculated for each discrete time step $t_{k}$. Discrete Kalman filter utilizes correction of previous estimation [29]. Consider step $k-1$ along with corresponding state vector estimation $\hat{\mathbf{x}}_{k-1}^{+}$and covariance matrix $\mathbf{P}_{k-1}^{+}$. The goal is to find state vector estimate for the next step $\hat{\mathbf{x}}_{k}^{+}$. First a priory estimation $\hat{\mathbf{x}}_{k}^{-}$is formed using straight mathematical model integration. It is corrected using sensor measurements vector $\mathbf{z}_{k}$ to obtain a posteriori estimation $\hat{\mathbf{x}}_{k}^{+}$. Covariance error matrix $\mathbf{P}_{k}^{-}$is also constructed from the previous step information using Riccati equation. It is then updated to $\mathbf{P}_{k}^{+}$ using measurements.

Kalman filter is designed for linear mathematical models and allows the best mean-square state vector estimation. It might be adapted for any non-linear mathematical models of both dynamical system and measurements,

$$
\begin{aligned}
\dot{\mathbf{x}}(t) & =\mathbf{f}(\mathbf{x}, t)+\mathbf{B u}(t)+\mathbf{G w}(t), \\
\mathbf{z}(t) & =\mathbf{h}(\mathbf{x}, t)+\mathbf{v}(t),
\end{aligned}
$$


where $\mathbf{w}(t)$ is a Gaussian dynamical model error with covariance matrix $\mathbf{D}, \mathbf{G}$ is a matrix of influence of model error on state vector, $\mathbf{v}(t)$ is a Gaussian measurements error with covariance matrix $\mathbf{R}$.

Kalman filter requires right-side functions $\mathbf{f}(\mathbf{x}, t)$ and $\mathbf{h}(\mathbf{x}, t)$ decomposition into the Taylor series in the vicinity of current state vector. Only linear terms are used in the filter. Dynamical system and measurements model matrices are

$$
\mathbf{F}_{k}=\left.\frac{\partial \mathbf{f}(\mathbf{x}, t)}{\partial \mathbf{x}}\right|_{\mathbf{x}=\hat{\mathbf{x}}_{\overline{\mathbf{x}}}, t=t_{k}}, \quad \mathbf{H}_{k}=\left.\frac{\partial \mathbf{h}(\mathbf{x}, t)}{\partial \mathbf{x}}\right|_{\mathbf{x}=\hat{\mathbf{x}}_{k}^{\bar{k}}, t=t_{k}} .
$$

Discrete extended Kalman filter uses non-linear dynamical and measurements models for a priory estimate prediction and a posteriori correction [30].

Prediction phase is

$$
\begin{aligned}
& \hat{\mathbf{x}}_{k}^{-}=\int_{t_{k-1}}^{t_{k}} \mathbf{f}(\mathbf{x}, t) d t, \\
& \mathbf{P}_{k}^{-}=\mathbf{\Phi}_{k} \mathbf{P}_{k-1}^{+} \mathbf{\Phi}_{k}^{\mathrm{T}}+\mathbf{Q}_{k},
\end{aligned}
$$

where $\mathbf{Q}_{k}$ is the covariance matrix of discrete-time process noise, it is calculated as

$$
\mathbf{Q}_{k}=\int_{t_{k-1}}^{t_{k}} \boldsymbol{\Phi}_{k} \mathbf{G D G}^{T} \boldsymbol{\Phi}_{k}^{T} d t .
$$

Correction phase is

$$
\begin{aligned}
& \mathbf{K}_{k}=\mathbf{P}_{k}^{-} \mathbf{H}_{k}^{\mathrm{T}}\left(\mathbf{H}_{k} \mathbf{P}_{k}^{-} \mathbf{H}_{k}^{\mathrm{T}}+\mathbf{R}_{k}\right)^{-1}, \\
& \hat{\mathbf{x}}_{k}^{+}=\hat{\mathbf{x}}_{k}^{-}+\mathbf{K}_{k}\left(\mathbf{z}_{k}-\mathbf{h}\left(\hat{\mathbf{x}}_{k}^{-}, t_{k}\right)\right), \\
& \mathbf{P}_{k}^{+}=\left(\mathbf{E}-\mathbf{K}_{k} \mathbf{H}_{k}\right) \mathbf{P}_{k}^{-},
\end{aligned}
$$

where $\mathbf{\Phi}_{k}=\exp \left(\mathbf{F}_{k}\left(t_{k}-t_{k-1}\right)\right)$ is a transition matrix between states $k-1$ and $k, \mathbf{E}$ is an identity matrix, $\mathbf{K}$ is a gain matrix.

\subsection{EKF application}

Consider the mock-up state vector as

$$
\mathbf{x}=\left[\begin{array}{llllllllll}
x & y & \varphi & q_{a} & q_{p} & \dot{x} & \dot{y} & \dot{\varphi} & \dot{q}_{a} & \dot{q}_{p}
\end{array}\right]^{T} .
$$

The developed mock-up motion model (6) is linear. Let the disturbances acting on the mock-up $\mathbf{w}(t)$ be a linear and angular accelerations. Then the matrix of influence of model error on state vector $\mathbf{G}$ from Eq. (11) is equal to matrix B (Eq. (6)).

Let the only measurements be a position and an angle of the mock-up body obtained by image processing of the video from the camera above the table (see Figure 1), then the measurement vector $\mathbf{z}=\left[\begin{array}{lll}x_{s} & y_{s} & \varphi\end{array}\right]^{T}$. In this case the measurement model Eq. (12) is derived from the Eq. (5): 


$$
\mathbf{z}=\left[\begin{array}{c}
\mathbf{r}+\frac{1}{m} \mathbf{T}_{d c m} \mathbf{A}\left(q_{p}-q_{a}\right) \\
\varphi
\end{array}\right],
$$

where

$$
\mathbf{T}_{d c m}=\left[\begin{array}{cc}
\cos \varphi & \sin \varphi \\
-\sin \varphi & \cos \varphi
\end{array}\right] .
$$

The measurement model Eq. (17) is not linear. The measurement matrix $\mathbf{H}$ Eq. (13) then is as follows

where

$$
\mathbf{H}=\left[\begin{array}{lll}
\mathbf{G}_{H} & \mathbf{A}_{H} & 0_{3 \times 5}
\end{array}\right],
$$

$$
\begin{aligned}
\mathbf{A}_{H} & =\frac{1}{m}\left[\begin{array}{cc}
A_{1} \cos \varphi+A_{2} \sin \varphi & -A_{1} \cos \varphi-A_{2} \sin \varphi \\
-A_{1} \sin \varphi+A_{2} \cos \varphi & A_{1} \sin \varphi-A_{2} \cos \varphi
\end{array}\right], \\
\mathbf{G}_{H} & =\left[\begin{array}{lll}
1 & 0 & \left(-A_{1} \sin \varphi+A_{2} \cos \varphi\right)\left(q_{p}-q_{a}\right) / m \\
0 & 1 & \left(-A_{1} \cos \varphi-A_{2} \sin \varphi\right)\left(q_{p}-q_{a}\right) / m \\
0 & 0 & 1
\end{array}\right] .
\end{aligned}
$$

\section{Path planning algorithm}

The path planning algorithm provides the trajectories from actual location to the desired target location. The most important issues to be addressed by the algorithms are fuel-optimality, collision avoidance with the target, robustness to perturbations. Also from the practical point of view, the path planning algorithm has to be real-time implementable on onboard computer, i.e. it should require low computation costs.

The motion equations of the mock-up with flexible elements are rather complicated even when the linear form is used and just one mode of flexible motion is considered. It is hard to develop fuel-optimal algorithm for trajectory generation and its onboard implementation could require high computational burden for real-time calculation. That is why in the paper considered the most simple approach that is similar to the one in [22]. However, it does not address the collision avoidance during docking, so the repelling potential is added which generates collision avoidance force when the mock-up approaches the target not in the front of docking side.

Consider a plane reference docking trajectory as a polynomial curve as

$$
\xi^{r e f}=\mathbf{b}_{0}+\mathbf{b}_{1} t+\mathbf{b}_{2} t^{2}+\mathbf{b}_{3} t^{3}+\mathbf{b}_{4} t^{4}
$$

where $\xi^{\text {ref }}=\left[x^{\text {ref }}, y^{\text {ref }}, \varphi^{\text {ref }}\right]^{T}$, vectors $\mathbf{b}_{i}$ are constant, determined by boundary conditions, i.e.

$$
\xi^{r e f}(t=0)=\xi_{0}, \dot{\xi}^{r e f}(t=0)=\dot{\xi}_{0}, \xi^{r e f}(t=T)=\xi_{T}, \dot{\xi}^{r e f}(t=T)=\dot{\xi}_{T}, \ddot{\xi}^{r e f}(t=T)=\ddot{\xi}_{T} .
$$

Here $T$ is the fixed time of docking maneuver, $\xi_{0}$ and $\dot{\xi}_{0}$ are the initial pose and velocity of the chaser mock-up, $\xi_{T}$ and $\dot{\xi}_{T}$ are the final pose and velocity of the mock- 
up when it docked to the target mock-up. Final acceleration $\ddot{\xi}_{T}$ must be set to achieve smooth docking trajectory at the final stage. Ones the boundary conditions are determined the vectors $\mathbf{b}_{i}$ are calculated as

$$
\begin{aligned}
& \mathbf{b}_{0}=\xi_{0}, \mathbf{b}_{1}=\dot{\xi}_{0}, \mathbf{b}_{2}=-\frac{1}{2 T^{2}}\left(12 \xi_{0}-12 \xi_{T}+6 \dot{\xi}_{0} T+6 \dot{\xi}_{T} T-\ddot{\xi}_{T} T^{2}\right), \\
& \mathbf{b}_{3}=\frac{1}{T^{3}}\left(8 \xi_{0}-8 \xi_{T}+3 \dot{\xi}_{0} T+5 \dot{\xi}_{T} T-\ddot{\xi}_{T} T^{2}\right), \\
& \mathbf{b}_{4}=\frac{1}{2 T^{4}}\left(6 \xi_{0}-6 \xi_{T}+2 \dot{\xi}_{0} T+4 \dot{\xi}_{T} T-\ddot{\xi}_{T} T^{2}\right) .
\end{aligned}
$$

Since the visual-based navigation system provides state vector of the mock-up body and not the docking system, final chaser mock-up position $\mathbf{r}_{T}^{c}$ and velocity $\dot{\mathbf{r}}_{T}^{c}$ must be calculated as

$$
\begin{aligned}
& \mathbf{r}_{T}^{c}=\mathbf{r}_{T}^{t}+\mathbf{A}_{d c m}\left(\varphi_{T}^{t}\right) \mathbf{r}_{d o c k}, \\
& \dot{\mathbf{r}}_{T}^{c}=\dot{\mathbf{r}}_{T}^{t}+\boldsymbol{\omega}_{T}^{t} \times\left(\mathbf{A}_{d c m}\left(\varphi_{T}^{t}\right) \mathbf{r}_{d o c k}\right),
\end{aligned}
$$

where $\mathbf{r}_{T}^{t}$ and $\dot{\mathbf{r}}_{T}^{t}$ are the target center of body position and velocity at time $t=T, \varphi_{T}^{t}$ and $\boldsymbol{\omega}_{T}^{t}$ are target angle and angular velocity at $t=T, \mathbf{r}_{\text {dock }}$ is the vector from the target center of body to the chaser center of body in docking position in mock-up reference frame $O_{t} x y, \mathbf{A}_{d c m}(\varphi)$ is the direction cosine matrix (see Figure 5).

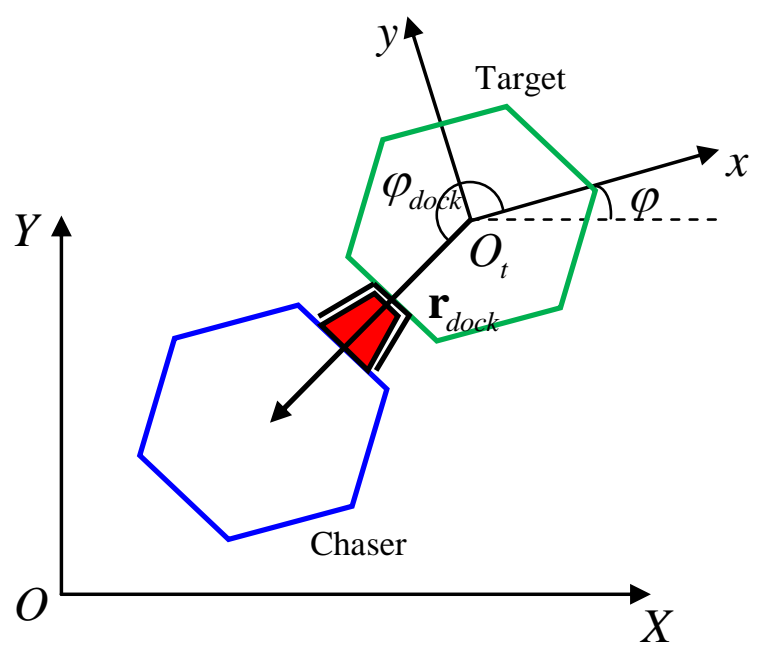

Figure 5. Scheme of two mock-ups docking

Calculate the state vectors of the non-cooperative target at the predefined time $T$. If its motion is non-controllable, then only the air table disturbances act on the mock-up. Using disturbance maps obtained by experimental study one can predict mock-up motion by integrating its motion equations.

The reference trajectory doesn't take into account collision avoidance restrictions. It means that reference trajectory even could pass through the target that 
leads to the crash of the mock-ups. To deal with the problem the specific repelling potential is developed. Its gradient produce the force that guide the mock-up center of body to the docking side. The potential $U_{p}$ has a form:

$$
U_{p}=k \exp \left(-\cos ^{2}\left(\frac{\alpha-\left(\varphi^{t}+\varphi_{\text {dock }}\right)}{2}\right)\right) \exp \left(-\tilde{k}_{r} \frac{r^{2}}{2}\right),
$$

where $\varphi^{t}$ is the attitude of the target mock-up, $\varphi_{\text {dock }}$ is the angle of the $\mathbf{r}_{\text {dock }}$ in the target-fixed reference frame (see Figure 6a), $r=\left|\mathbf{r}^{t}-\mathbf{r}^{c}\right|=\sqrt{x^{2}+y^{2}}, \alpha=\operatorname{atan}(y / x)$, $k, \tilde{k}_{r}$ are the positive constants. Set $k=2 \mathrm{~N} \cdot \mathrm{m}, \tilde{k}_{r}=0.1 \mathrm{~m}^{-2}$ and $\varphi^{t}+\varphi_{\text {dock }}=0$, then the potential function has the form, presented in Figure 8a. The collision avoidance forces calculated as $\mathbf{F}=-\operatorname{grad}\left(U_{p}\right)$ are shown in Figure $6 \mathrm{~b}$. So, there is no collision forces from the docking side of the mock-up.

a)

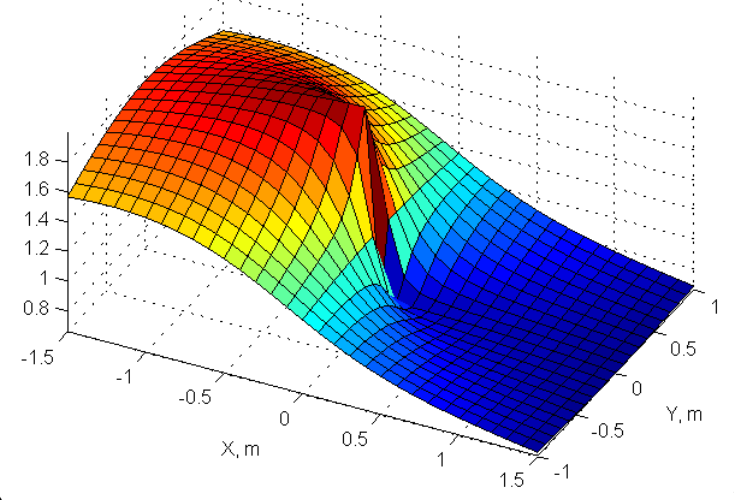

b)

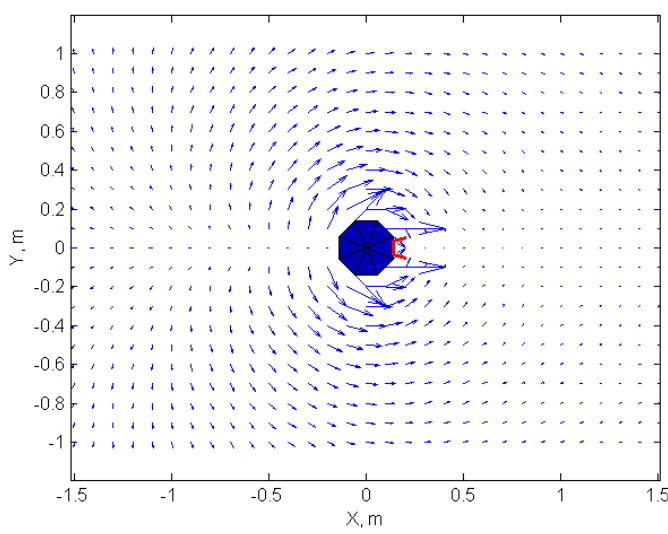

Figure 6. Collision avoidance potential (a) and forces (b)

\section{The laboratory simulations}

Consider the mock-up with flexible rods motion on the test bench during the docking to the noncooperative target mock-up. Main parameters of the mock-up with flexible rods and measurement system are presented in Table 1 . Some of them were obtained by processing measurements of mock-up motion during preliminary experiments using least square method.

The logic of the whole system work is presented in Figure 7. First, when the time of the docking maneuver $T$ is specified, the EKF estimates the state vectors of both the chaser and target mock-ups, and based on these estimates the reference trajectory for docking is calculated. Then, the reference trajectory and state vector estimates are used for calculation of control that consists of reference trajectory tracking part with taking into account flexible motion and collision avoidance terms. The resulting control vector implemented by the fan thrusters. Due to the unaccounted disturbances and collision avoidance control the actual mock-up trajectory deviates from reference. That is why it is necessary update the reference trajectory with some timestep $\Delta t$. 
Table 1. Parameters of the simulation

\begin{tabular}{l|c}
\hline \multicolumn{2}{c}{ Dynamical characteristics of the mock-up } \\
\hline Mass of the whole mock-up, $m$ & $5.2 \mathrm{~kg}$ \\
\hline Mass of the flexible rod, $m_{a}=m_{p}$ & $0.3 \mathrm{~kg}$ \\
\hline Flexible rod length, $L$ & $1.2 \mathrm{~m}$ \\
\hline Mock-up body moment of inertia, $J_{s}$ & $0.05 \mathrm{~kg} \cdot \mathrm{m}^{2}$ \\
\hline $\begin{array}{l}\text { Mock-up with rods moment of iner- } \\
\text { tia, } J\end{array}$ & $0.15 \mathrm{~kg} \cdot \mathrm{m}^{2}$ \\
\hline Natural main frequency, $\omega_{0}$ & $1.5 \mathrm{~Hz}$ \\
\hline Rod displacement vector, & {$[0.001 ; 0.423]$} \\
A $=\left[A_{1} ; A_{2}\right]$ & \\
\hline \multicolumn{2}{c}{ Control system parameters } \\
\hline Maximum ventilator thrust, $\tau$ & $0.95 \mathrm{~N}$ \\
\hline Maximum control force, $F_{O}$ & $1.9 \mathrm{~N}$ \\
\hline Maximum control torque, $T_{s}$ & $0.4 \mathrm{~N} \cdot \mathrm{m}$ \\
\hline \multicolumn{1}{c}{ Control algorithms parameters } \\
\hline $\begin{array}{l}\text { Mean square position measurements } \\
\text { errors, } \sigma_{r}\end{array}$ & $25 \mathrm{~mm}$ \\
\hline $\begin{array}{l}\text { Mean square angle measurements } \\
\text { error, } \sigma_{\varphi}\end{array}$ & $0.1 \mathrm{deg}$ \\
\hline Control parameter, $k_{r}$ & $10 \mathrm{~s}^{-1}$ \\
\hline Control parameter, $k_{v}$ & $0.62 \mathrm{~s} \mathrm{~s}^{-1}$ \\
\hline Control parameter, $k_{\varphi}$ & $k_{\omega}$ \\
\hline Control parameter, $k_{\omega}$ & \\
\hline
\end{tabular}




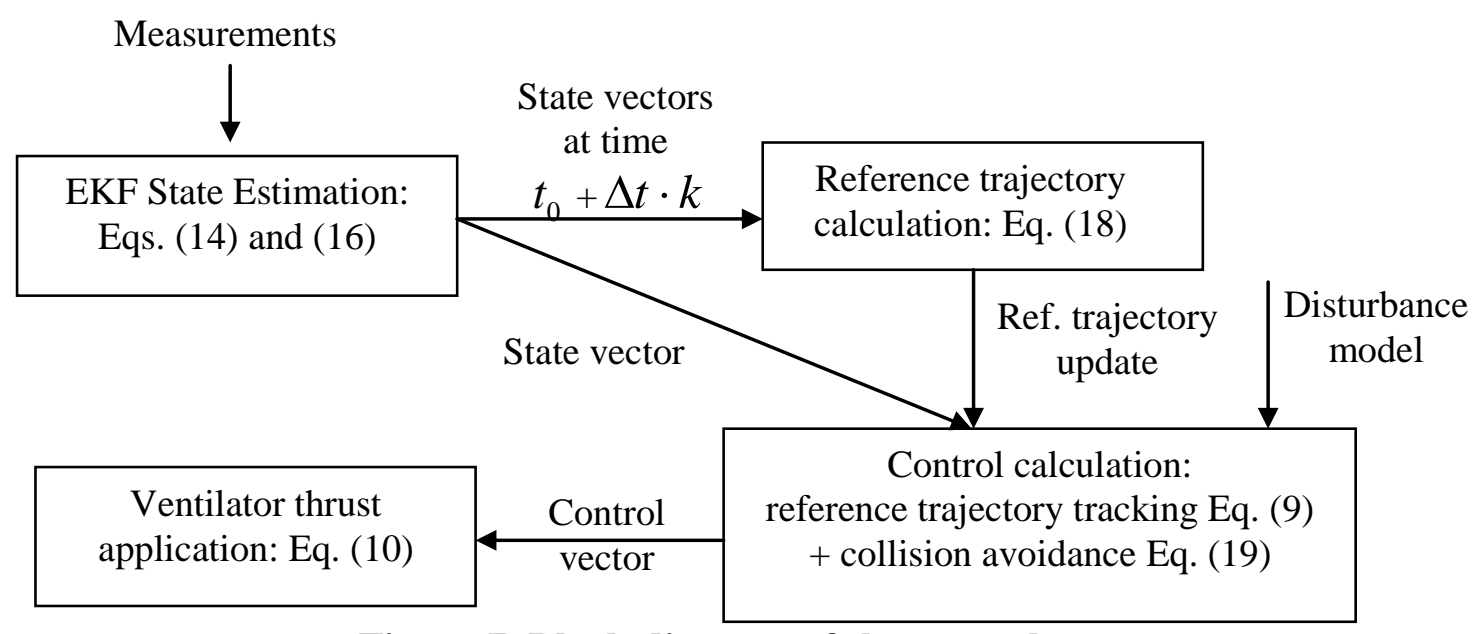

Figure 7. Block diagram of the control system

Consider for the docking with the fixed target mock-up with non-cooperative orientation. Initially the flexible motion of the chaser mock-up is manually excited. Set the time for docking maneuver to $20 \mathrm{~s}$. First, let the control law disregard the rods vibrations and be a simple PD-controller for reference trajectory tracking. Figure 8 shows the motion of the chaser mock-up with flexible rods to the target, a set of snapshots are presented. Estimated by EKF deviations of the main low flexible mode of the both rods are not dumped, but even became slightly more exited during the motion (Figure 9). The flexible motion leads to the mock-up body deviation from the reference trajectory. It causes the error in position at the final stage of docking of about 2-3 cm (Figure 10). For our docking system the error must not be more that 2 $\mathrm{cm}$, so the docking occasionally fails because of the mock-up body deviations from the reference trajectory, it is unacceptable. The control vector dependence on time is presented in Figure 11. As one can see, because of the flexible motion of the roods, the implemented control are saturated most of the time.

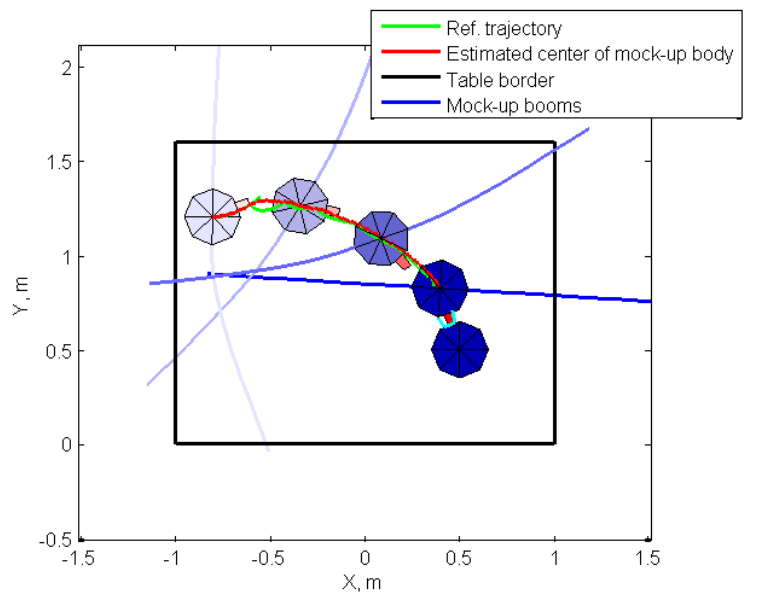

Figure 8. Docking with PD-control disregarding flexible motion

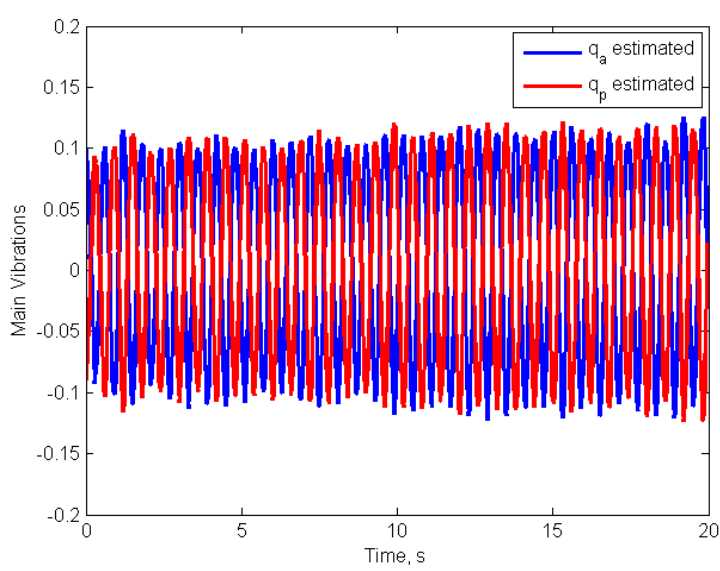

Figure 9. Main vibrations dependence on time 


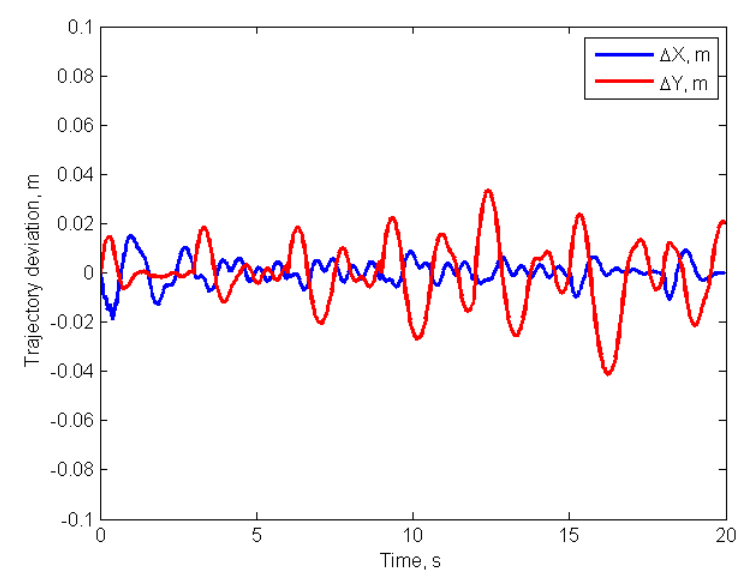

Figure 10. Deviation from reference trajectory

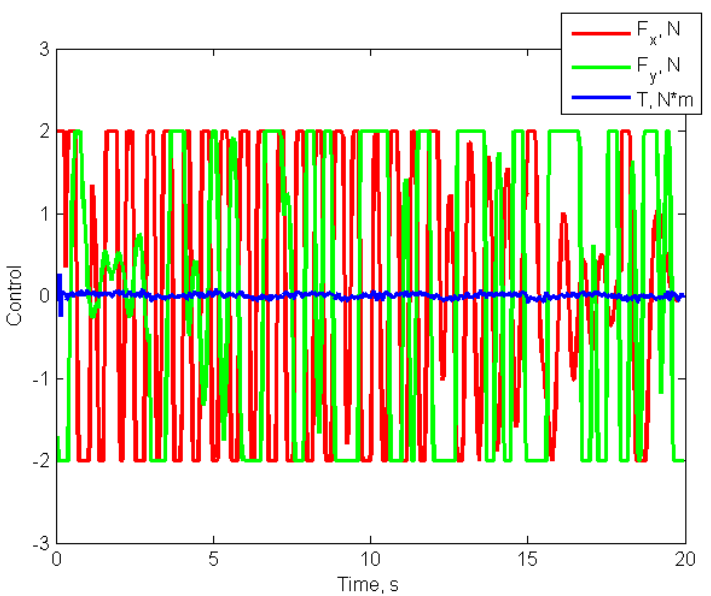

Figure 11. Control vector dependence on time

Lets apply the developed control law (9) for almost the same initial conditions of the mock-ups. Figure 12 shows the chaser mock-up motion. During the maneuver the flexible motion is significantly damped (Figure 13). The deviation of the mock-up body from reference trajectory is almost $4 \mathrm{~cm}$ in the beginning, but closer to the target mock-up the smaller deviation. And finally due to flexible motion damping the error is about $1 \mathrm{~cm}$ (Figure 14). The control forces and torque is presented in Figure 15. In the beginning the implemented control is also saturated, but in the end the calculated control executed as it is.

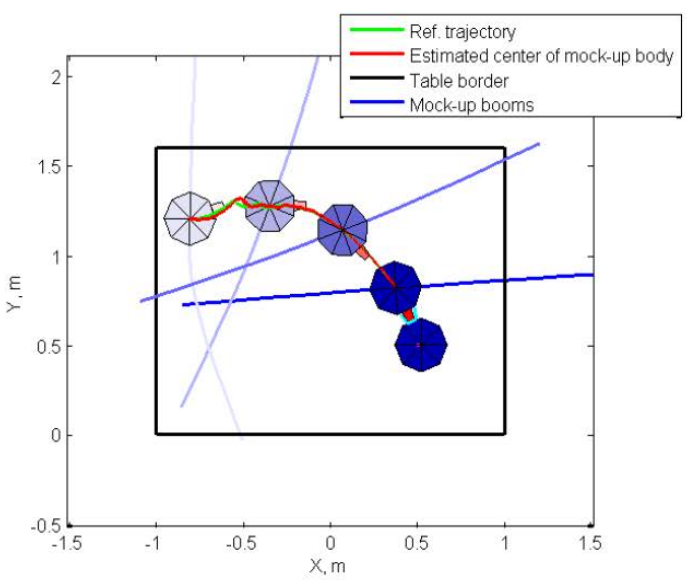

Figure 12. Docking with control taking into account flexible motion

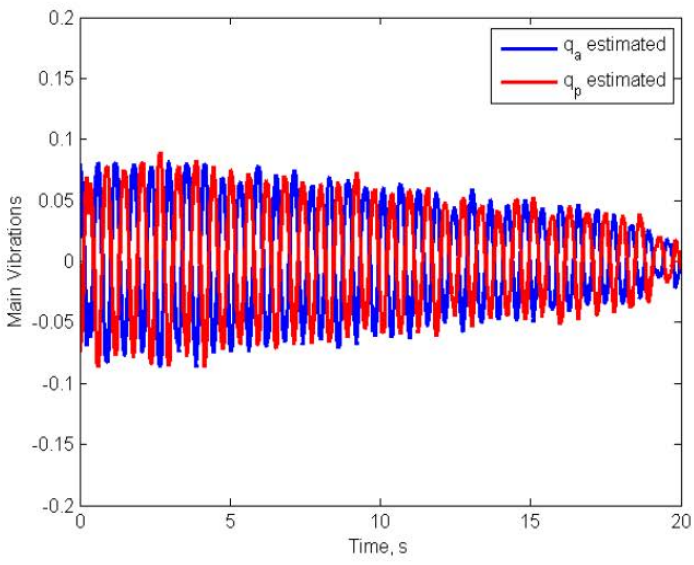

Figure 13. Main vibrations dependence on time 


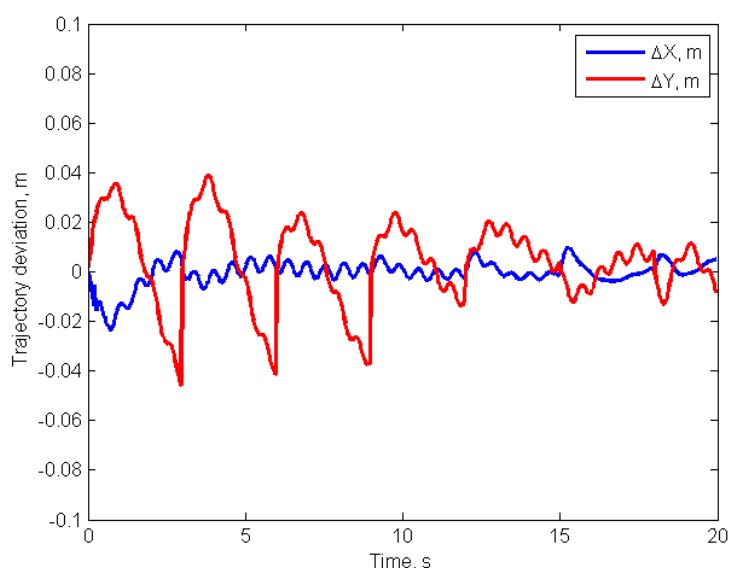

Figure 14. Deviation from reference trajectory

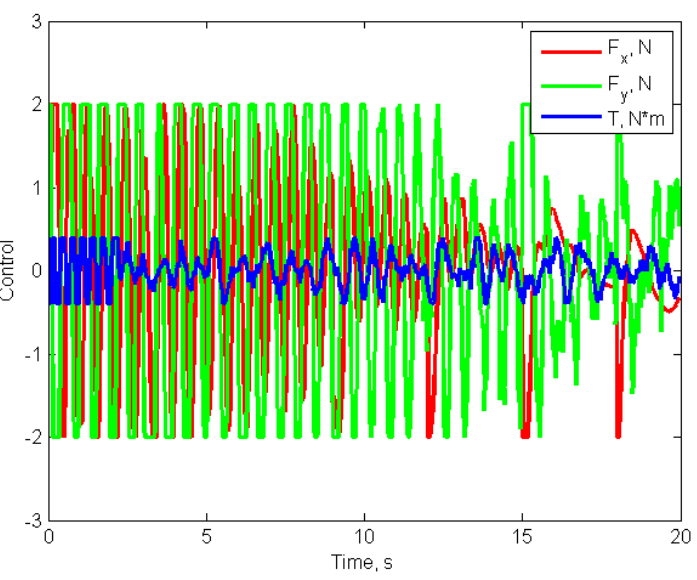

Figure 15. Control vector dependence on time

Now consider the influence of the collision avoidance addition in control on the motion during the docking. Let's place the target mock-up in the middle of the table and turn it docking side away from the direction to the chaser. Since the path planning algorithm is not taking into account the collision avoidance terms, the reference trajectory could lead directly to the mock-ups crashing. Figure 16 present that situation. Then if we add the collision avoidance potential Eq. (19) to the control, the mock-up deviates from the reference trajectory to avoid the crash as shown in Figure 17. When the mock-up is moving from the docking side of the target, the additional control force is almost zero (see Figure 6b). If the deviation from the reference docking trajectory is significant it is necessary to update it by recalculating the coefficients in Eq. (18). The updates could be also regular with some time step.

Finally, when the target mock-up moves freely along the table it is possible to predict its pose by integration its motion equations. So, the predicted state vector is used for reference trajectory calculation. But some disturbances remain unaccounted, that is why the reference trajectory has to be regularly updated.

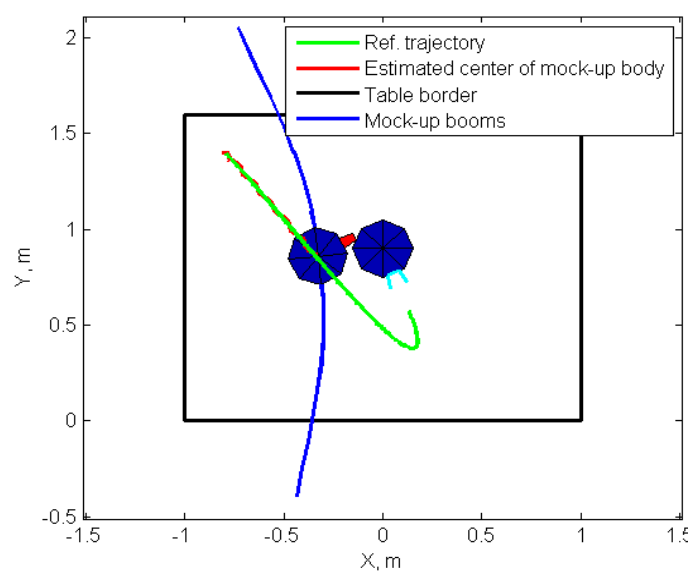

Figure 16. Mock-ups crash

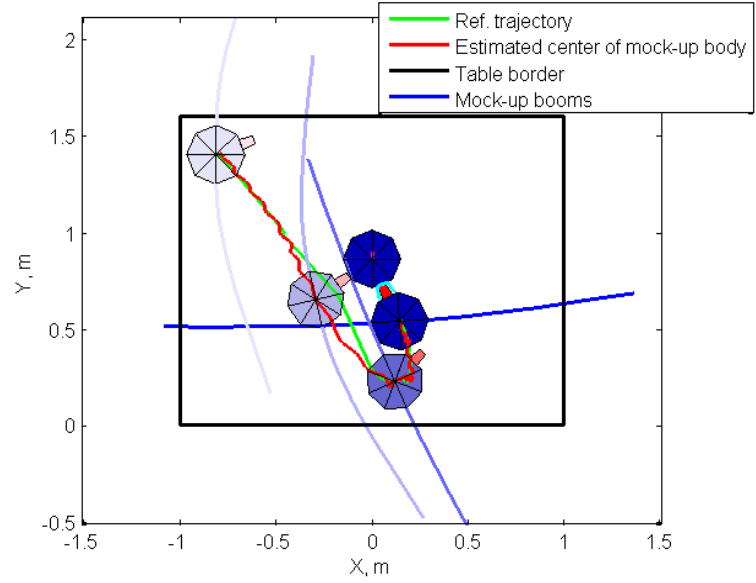

Figure 17. Collision avoidance demonstration 


\section{Conclusion}

The developed control algorithm of the mock-up with flexible rods is able to diminish the vibrations and track the mock-up body docking trajectory with the required accuracy. The experimental study showed that it works well even with restrictions in control vector. The algorithm is suitable for implementation for on-board computer with real-time requirements, though it is necessary to set the parameters of the flexible elements. Moreover, the assumptions about one natural flexible mode with low frequency dominating and about smallness rod deviations from equilibrium position must be satisfied.

\section{References}

1. Izzo D., Pettazzi L. Autonomous and Distributed Motion Planning for Satellite Swarm // J. Guid. Control. Dyn. 2007. Vol. 30, № 2. P. 449-459.

2. D'Arrigo P., Santandrea S. "The APIES Mission" ASTRIUM Ltd./ESAESTEC Feasibility Study A0/1-3846/02/NL/JA, Stevenage, U.K., 2004.

3. Folkner W.M., Hellings R.W., Maleki L. LISA Laser Interferometer Space Antenna Fof Gravitational Wave Measurements // Aerosp. Sci. Meet. Exhib. 33rd, Reno, NV, Jan 9-12, 1995.

4. Rybus T., Seweryn K. Planar air-bearing microgravity simulators: Review of applications, existing solutions and design parameters // Acta Astronaut. 2016. Vol. 120. P. 239-259.

5. Segal S., Carmi A., Gurfil P. Stereovision-Based Estimation of Relative Dynamics Between Noncooperative Satellites: Theory and Experiments // IEEE Trans. Control Syst. Technol. 2014. Vol. 22, № 2. P. 568-584.

6. Ivanov D.S. et al. Laboratory Facility For Microsatellite Mock-Ups Motion Control Verification // J. Comput. Syst. Sci. Int. 2017.

7. Rybus $\mathrm{T}$. et al. New planar air-bearing microgravity simulator for verification of space robotics numerical simulations and control algorithms // 12th ESA Symp. Adv. Sp. Technol. Robot. Autom. 2013. Vol. 1, № 1. P. 1-8.

8. Jorgensen G., Bains E. SRMS history, evolution and lessons learned // Proc. AIAA Sp. Conf. Expo. Long Beach. 2011. P. 24.

9. Bilton A. et al. Sensor architecture for the robotic control of large flexible space structures // J. F. Robot. 2007. Vol. 24, № 4. P. 297-310.

10. Sabatini M. et al. Analysis and experiments for delay compensation in attitude control of flexible spacecraft // Acta Astronaut. Elsevier, 2014. Vol. 104, № 1. P. 276-292. 
11. Gasbarri P., Pisculli A. Dynamic / control interactions between flexible orbiting space-robot during grasping, docking and post-docking // Acta Astronaut. Elsevier, 2015. Vol. 110. P. 225-238.

12. Hartley E.N. et al. Model predictive control system design and implementation for spacecraft rendezvous // Control Eng. Pract. 2012. Vol. 20, № 7. P. 695-713.

13. Breger L.S., How J.P. Safe Trajectories for Autonomous Rendezvous of Spacecraft // J. Guid. Control. Dyn. 2008. Vol. 31, № 5. P. 1478-1489.

14. Liu X., Lu P. Solving Nonconvex Optimal Control Problems by Convex Optimization // J. Guid. Control. Dyn. American Institute of Aeronautics and Astronautics, 2014. Vol. 37, № 3. P. 750-765.

15. Lu P., Liu X. Autonomous Trajectory Planning for Rendezvous and Proximity Operations by Conic Optimization // J. Guid. Control. Dyn. American Institute of Aeronautics and Astronautics, 2013. Vol. 36, № 2. P. 375-389.

16. Ventura J. et al. Fast and Near-Optimal Guidance for Docking to Uncontrolled Spacecraft // J. Guid. Control. Dyn. 2016. P. 1-17.

17. Kobilarov M., Pellegrino S. Trajectory Planning for CubeSat Short-Time-Scale Proximity Operations // J. Guid. Control. Dyn. American Institute of Aeronautics and Astronautics, 2014. Vol. 37, № 2. P. 566-579.

18. Ciarci M., Grompone A., Romano M. A near-optimal guidance for cooperative docking maneuvers // Acta Astronaut. Elsevier, 2014. Vol. 102. P. 367-377.

19. Pearson J. The glideslope approach // Adv. Astronaut. Sci. 1989. Vol. 69. P. 109-123.

20. Nolet S. Development of a guidance, navigation and control architecture and validation process enabling autonomous docking to a tumbling satellite // Ph.D. Diss. Dept. Aeronaut. Astronaut. Inst. Technol. Cambridge, MA. 2007. 322 p.

21. Guglieri G. et al. Design and development of guidance navigation and control algorithms for spacecraft rendezvous and docking experimentation // Acta Astronaut. 2014. Vol. 94, № 1. P. 395-408.

22. Sabatini M., Palmerini G.B., Gasbarri P. A testbed for visual based navigation and control during space rendezvous operations // Acta Astronaut. Elsevier, 2015. Vol. 117. P. 184-196.

23. SputniX - Orbicraft [Electronic resource]. URL: http://sputnix.ru/ru/products/eduru/orbikraft-1-0 (accessed: 02.12.2016).

24. Koptev M., Proshunin N., Ivanov D. Motion Determination of Microsatellite Control System Mock-Ups on Aerody-namic Testbed Using Monocular Vision // Prepr. KIAM RAS. 2015. № 109. P. 32.

25. MEIROVITCH L., QUINN R.D. Equations of motion for maneuvering flexible spacecraft // J. Guid. Control. Dyn. 1987. Vol. 10, № 5. P. 453-465. 
26. Santini P., Gasbarri P. Dynamics of multibody systems in space environment; Lagrangian vs. Eulerian approach // Acta Astronaut. 2004. Vol. 54, № 1. P. 124.

27. Ovchinnikov My. et al. Angular motion equations for a satellite with hinged flexible solar panel // Acta Astronaut. 2016. Vol. 128. P. 534-539.

28. Ivanov D., Ovchinnikov M., Tkachev S. Attitude control of a rigid body suspended by string with the use of ventilator engines // J. Comput. Syst. Sci. Int. 2011. Vol. 50, № 1. P. 104-116.

29. Kalman R.E., Bucy R.S. New Results in Linear Filtering and Prediction Theory // Trans. ASME, Ser. D, J. Basic Eng. 1961. Vol. 83. P. 95-108.

30. Wertz J.R. Spacecraft Attitude Determination and Control. Dordrecht/Boston, London: Acad. press, 1990. 880 p. 


\section{Content}

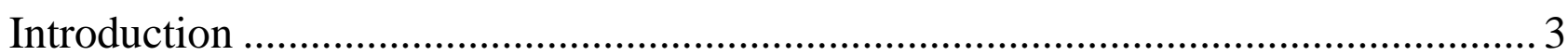

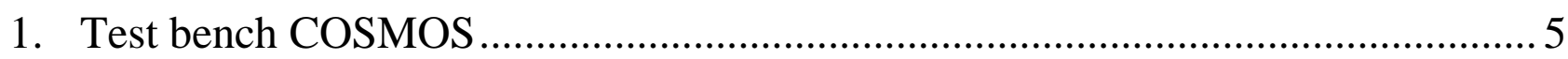

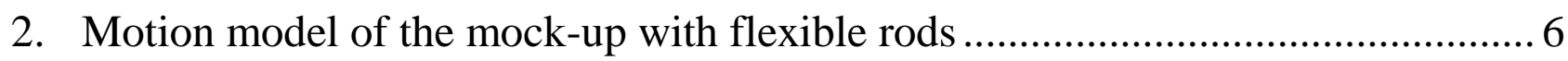

2.1. Mock-up motion equations derivation ................................................. 7

2.2. Control Synthesis ................................................................................. 10

2.3. Control Realization by Fans ............................................................... 11

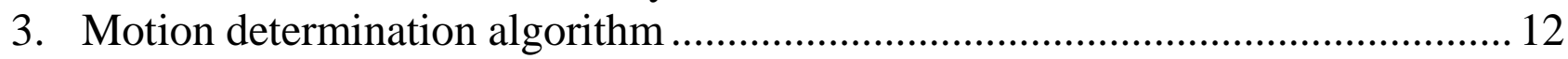

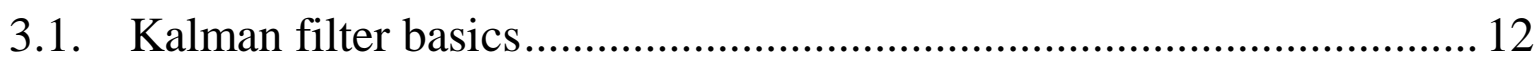

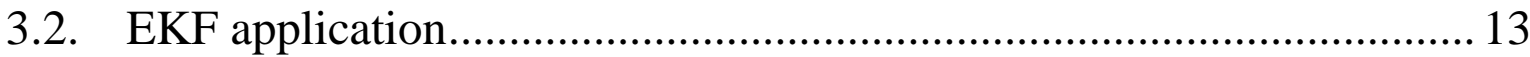

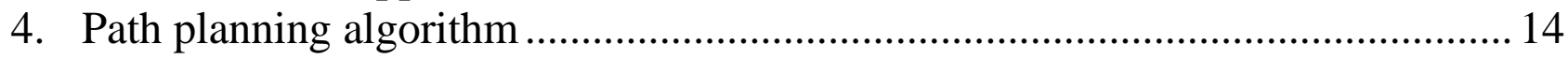

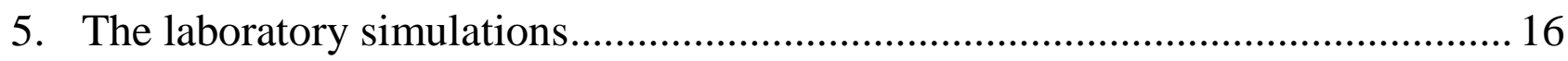

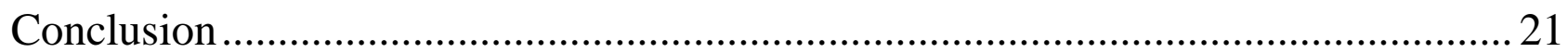

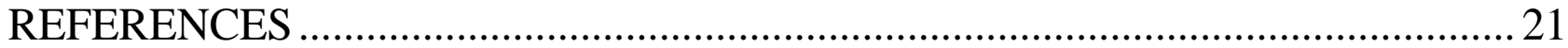

